\section{"Dip and Dry" Coating Process Developed for Use of SWNTs in Wearable Electronics}

Wearable electronics is an emerging class of materials for applications such as embedded health-monitoring devices, wearable displays, and portable power for personal electronic devices. Nanoelectronics must somehow be combined with textiles to achieve lightweight, flexible, and stretchable fabrics. Recently, Y. Cui and a team of researchers at the Department of Materials Science and Engineering, and the Department of Electrical Engineering, Stanford University, used a simple "dip and dry" process with single-walled carbon nanotube (SWNT) ink to transform common textiles into wearable power devices.

As reported in the online January 5, 2010 issue of Nano Letters (DOI: 10.1021/ n1903949m), Cui and co-researchers dipped a fabric sheet (either 100\% cotton or woven polyester) into a black, SWNT ink (prepared by dispersing SWNTs in water with a surfactant) and then dried the fabric at $120^{\circ} \mathrm{C}$ for $10 \mathrm{~min}$. After SWNT coating, the textiles retained their original textures but displayed high conductivity, with a sheet resistance of
$4 \Omega /$ sq for the cotton fabric. Conductivity for the polyester fabric is lower because SWNT adsorptivity is lower. However, conductivity was shown to increase by repeating dipping in the SWNT ink. Scanning electron microscopy showed conformal coating of the SWNTs onto the fabrics' fibers, which the researchers said is a result of a combination of the mechanical flexibility of individual SWNTs and the strong binding energy between the SWNTs and the fibers. Transmission electron microscopy of the SWNT-coated cotton fabric showed that the SWNTs formed cross-linked networks that provide conducting pathways.

Standard industry tests using adhesive tape and washing in water (with subsequent squeezing and wringing) resulted in no observable SWNTs on the tape or in the water. In contrast to previously reported stretchable conductors, whose conductance decreased with stretching, conductance of the SWNT-treated fabric increased until the fabric was stretched to 2.4 times its original length, which the researchers said was due to the improvement of the mechanical contacts between fibers that leads to better electrical contacts between SWNTS. Galvanostatic cycling was used to demonstrate that the specific capacitance of electrodes made from SWNT-treated cotton fabric is 2-3 times higher than SWNTs on a plastic substrate and comparable to the highest values reported for SWNT electrode materials. The areal capacitance of $0.48 \mathrm{~F} / \mathrm{cm}^{2}$ reported for the SWNT-treated fabric, however, is the highest value reported for a SWNT device and is comparable to capacitances available commercially. The researchers said that this is due to the high mass loading of their material. The supercapacitor device displayed only a $2 \%$ variation in capacitance over 130,000 cycles. In addition, there was no change in specific capacitance after stretching. The researchers said, "With lightweight and cheap cotton or fabrics and the existing textile fabrication infrastructure, largescale energy storage with low material and processing costs is also feasible. When combined with inks of other functional materials, other types of electronics could be created, such as wearable solar cells and batteries. These porous conductors with excellent properties will find a wide range of applications."

StEVEn TROHALAKI

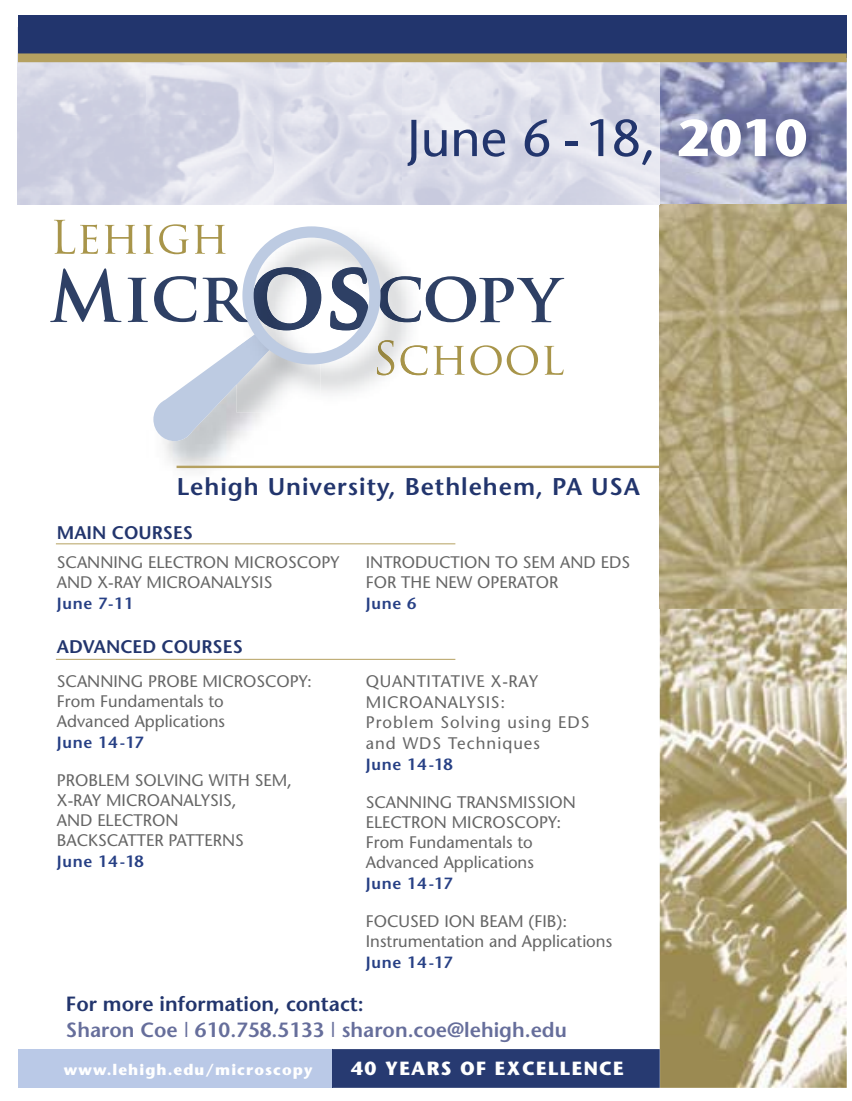

\section{JANIS}

\section{Cryogen Free Probe Stations}

- $<5 \mathrm{~K}-650 \mathrm{~K}$ cryocooler based systems, using no liquid cryogens

Fully vibration isolated sample

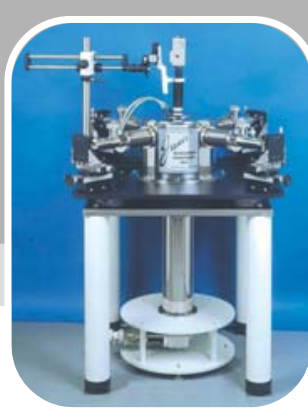
mounting stage for sub-micron stability

Two to six probes, $\mathrm{DC}$ to $67 \mathrm{GHz}$ frequency range, with fiber optic option

Zoom optics with camera, monitor and full system integration

Complete packages including probe station, temperature controller, optics, and vacuum pump

- LHe and $\mathrm{LN}_{2}$ cooled, and room temperature probe stations also available

\section{Janis Research Company}

2 Jewel Drive Wilmington, MA 01887 USA TEL +1 978 657-8750 FAX +1 978 658-0349 sales@janis.com Visit our website at WWW.janis.com 